\title{
Percutaneous Cannulated Screws with Tension Band Wiring Technique in Patella Fractures
}

\author{
Jin-Ho Cho, MD
}

Department of Orthopaedic Surgery, Inje University Ilsan Paik Hospital, Goyang, Korea

\begin{abstract}
Introduction: Most patellar fractures are transverse involving the central third. Open reduction and stabilization of transverse patellar fractures is indicated if there is more than $2-3 \mathrm{~mm}$ of fragment separation and/or articular incongruity.

Surgical Technique: This study describes a percutaneous 2 cannulated screws and modified tension band wiring technique to treat transverse patellar fractures.

Materials and Methods: We performed 30 cases of displaced transverse patellar fractures with this technique. The clinical outcomes of these patients were evaluated with simple radiographs, range of motion and Lyshom score.

Results: This technique has shown to provide satisfactory clinical results and excellent knee functions.

Conclusions: This technique provide stable fixation, allows early motion exercise by minimizing injury to extensor mechanism and reduce cosmetic problem in scar.
\end{abstract}

Keywords: Patella, Fracture, Percutaneous surgery, Cannulated screw, Modified tension band technique

\section{Introduction}

Patella fractures account for $1 \%$ of all musculoskeletal system fractures ${ }^{1)}$. Most fractures of indirect injury are transverse involving the middle third of the patella. Conservative treatment is limited to fractures with minimal displacement and those with no significant incongruity of the articular surface, while surgical treatment is recommended when fragment displacement is 2-3 $\mathrm{mm}$ or when there is articular incongruency ${ }^{2)}$.

The objectives of surgical treatment include preservation of the knee cap to the greatest possible extent, precise anatomic reduction of the joint surface by stable fixation, and restoration of the

Received April 28, 2013; Revised (1st) August 2, 2013; (2nd) September 2, 2013; Accepted September 3, 2013

Correspondence to: Jin-Ho Cho, MD

Department of Orthopaedic Surgery, Inje University Ilsan Paik Hospital, 170 Juhwa-ro, Ilsanseo-gu, Goyang 411-706, Korea

Tel: +82-31-910-9733, Fax: +82-31-910-7967

E-mail: osd07@paik.ac.kr

This is an Open Access article distributed under the terms of the Creative Commons Attribution Non-Commercial License (http://creativecommons.org/licenses/by-nc/3.0/) which permits unrestricted non-commercial use, distribution, and reproduction in any medium, provided the original work is properly cited. knee-extensor mechanism, thus allowing early mobilization ${ }^{3,4)}$. The usual surgical intervention is carried out through a long skin incision with a substantial soft tissues dissection to sufficient for fracture and joint-surface visualization. Wide surgical incisions are associated with a higher probability of postoperative adhesions ${ }^{5}$, prolonged disability for work, as well as a cosmetically unsightly scar ${ }^{6}$. The presence of lacerations or abrasions around the operative site may delay the surgery until the danger of contaminating the operative wound is minimal ${ }^{7}$.

In contrast, percutaneous surgical techniques preserve the vascular supply of patellar fragments, which may improve fracture consolidation. However, this treatment is technically demanding and not applicable to all fracture patterns ${ }^{8}$. This technique combines the advantages of minimal invasive surgery and rigid internal fixation, thereby permitting early rehabilitation and good functional outcome.

\section{Surgical Technique}

Under either general anaesthesia or spinal or epidural analgesia, the patients were placed in a supine position on a standard radiolucent operating table with the knee in full extension. Pneumatic tourniquet was applied to the upper thigh and inflated to control 

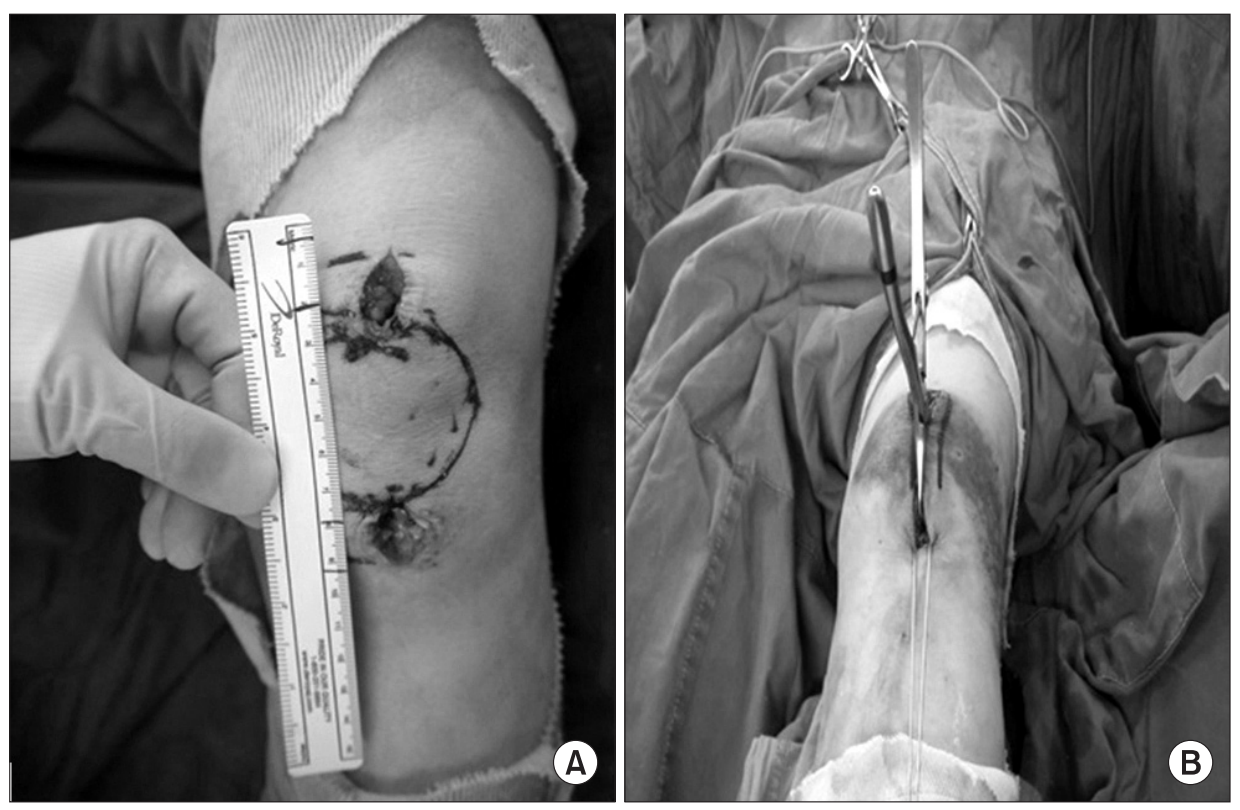

Fig. 1. (A) Photograph showed 2 minimal $15 \mathrm{~mm}$ skin incisions were made on patellar upper and lower pole area on left knee. (B) Fracture was reduced by manipulation and maintained by a patellar reduction clamp applied through skin incisions percutaneously. Thereafter, two Kirschner wires were drilled longitudinally perpendicular to the fracture line from lower pole to upper pole of patella on left knee.
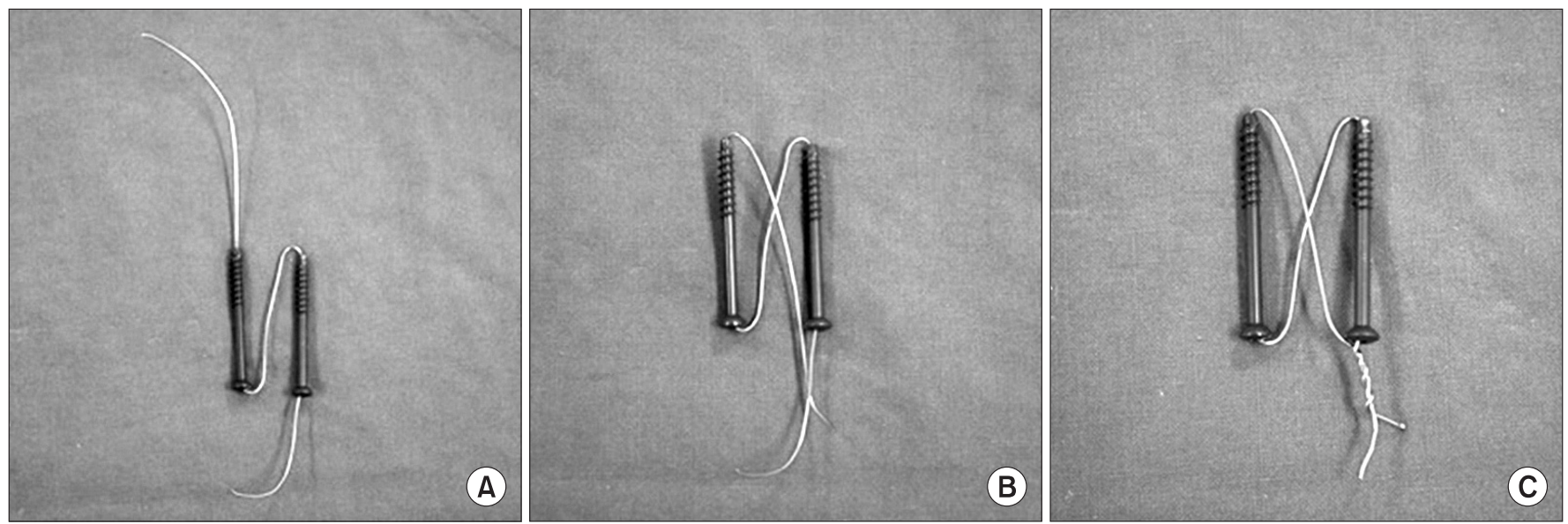

Fig. 2. (A) Schematic photograph showed rolled wire was inserted into lateral cannulated screw from head side to tail side and then pulled out percutaneously toward medial screw head side. At second, this wire tip inserted into medial screw from head to tail side. (B) And then pulled out percutanously toward lateral screw head side. (C) Wire's tips were twisted between both screw's head sides in a "figure of butterfly" configuration.

bleeding during the procedure. At first, 2 minimal $15-20 \mathrm{~mm}$ skin incisions were made on patellar upper and lower pole area (Fig. 1A). Through two skin incision, entrapped soft tissue or hematoma were removed with curette. Under C-arm image intensifier control, the upper pole of the proximal fragment and the lower pole of the distal fragment were identified by palpation and then confirmed with fluoroscopy and then the fracture was anatomically reduced by manipulation and maintained by a patellar reduction clamp applied through skin incisions percutaneously. Thereafter, two Kirschner wires (K-wires) were drilled longitudinally perpendicular to the fracture line from lower pole to upper pole of patella (Fig. 1B). And then adequate sized two half threaded $4.0 \mathrm{~mm}$ cannulated screws (Solco Biomedical Co., Pyeongtaek, Korea) inserted through the K-wire. Next step is modified tension band wiring through screw's cannals. At first, 0.97 $\mathrm{mm}$ (18G, No7) rolled wire was inserted into lateral cannulated screw from head side to tail side and then pulled out percutaneously toward medial screw head side. At second, this wire tip inserted into medial screw from head to tail side (Fig. 2A) and then pulled out percutanously toward lateral screw head side (Fig. 2B). Finally, wire's tips were twisted between both screw's head sides. A wire was then placed in a "figure of butterfly" configuration shape with cannulated screws (Fig. 2C). Stability of reduction and fixation was checked with image intensifier by flexing and ex- 

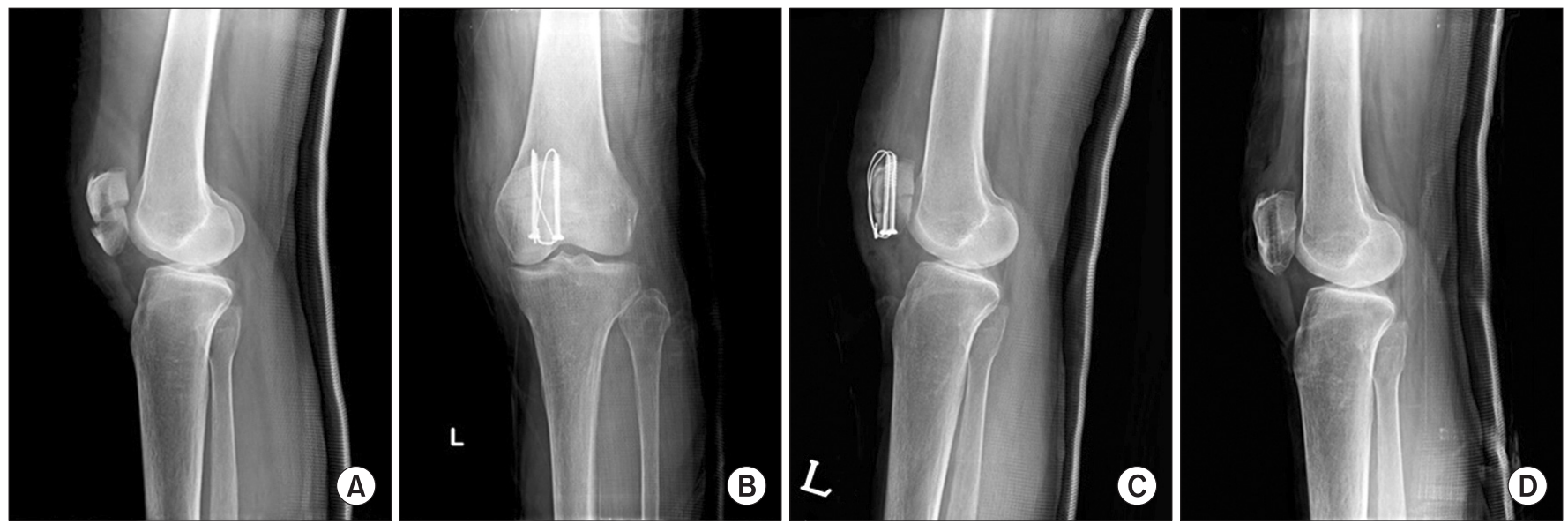

Fig. 3. Simple radiograph of 60-year-old woman patient with transverse patellar fracture in her left knee by slip down injury. (A) Preoperative lateral radiograph. (B, C) Immediate postoperative radiographs. (D) After metal removal simple radiograph at the 1-year follow-up.

tending the knee. After routine suture of subcutaneous tissue and skin, cylinder splint applied with extended knee position fully.

The suction drain was removed 24-48 hours after the surgery and all the patients were discharged home with an adjustable angle hinged knee brace allowing initial range of motion (ROM) of $0^{\circ}-40^{\circ}$. The flexion angle was increased $15^{\circ}-20^{\circ}$ weekly, with the objective of full flexion at 6 weeks. Quadriceps setting exercises, active knee extension together with gentle active ROM exercises to be increased sequentially, were encouraged immediately after the operation but quadriceps exercises against resistance were delayed for 6 weeks. All patients were encouraged to ambulate with crutches without any weight bearing on the injured limb. Partial weight bearing was allowed 3-4 weeks postoperatively. Full weight bearing was allowed after the fracture was healed radiographically.

\section{Materials and Methods}

Between December 2009 and January 2012, we performed the decribed technique on 30 patients. Patients were an average of 45 years of age (range, 21 to 86 years). Mean follow-up period was 15 months (range, 12 to 25 months). Causes of fractures were 18 cases in slip down and 12 cases in traffic accident. The clinical outcomes of these patients were evaluated with simple radiographs, ROM and Lyshom score. They returned to exercise after 6 months postoperatively.

\section{Results}

All cases were gained complete bone union (Fig. 3). Radiographic bone union period were 7.3 weeks (range, 6 to 11 weeks).

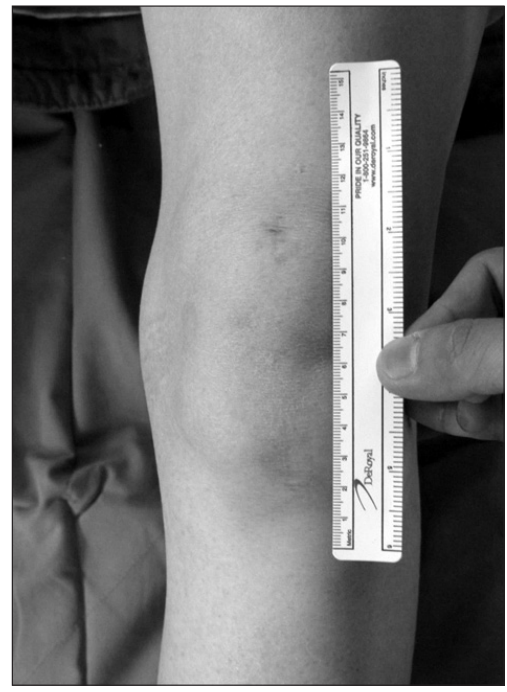

Fig. 4. Photograph showed minimal skin incision scar at the 1-year follow-up.

ROM of knee was flexion contracture $2^{\circ}$ (range, $0^{\circ}$ to $5^{\circ}$ ) and further flexion were $133^{\circ}$ (range, $125^{\circ}$ to $135^{\circ}$ ). Lysholm knee score were 93 (range, 85 to 97). We have no complications like limitation of knee motion, loss of reduction, failure or migration of hardware and infection at last follow up. We also able to reduced cosmetic problem in scar at last follow up (Fig. 4).

\section{Discussion}

There are two major mechanisms of injury in patella fractures: direct and indirect trauma. For example, the patella may be fractured by a direct blow to the knee during a fall or when it hits the dashboard in a traffic accident. Treatment options for patellar 
fractures depends on the type of fracture, size of the fragments, stability of the extensor mechanism, and regularity of the articular surface ${ }^{9-11}$. Surgery is indicated if there is damage to the extensor mechanism or in fractures associated with $2 \mathrm{~mm}$ step-off incongruity $^{9,10)}$. The main goal of the treatment of patella fractures is to provide a regular articular surface, to maintain rigid fixation, and to preserve joint function by initiating early knee range of motion ${ }^{9,10)}$. Displaced transverse fractures of the patella are commonly treated by internal fixation. The widely accepted surgical technique is open reduction and the modified tension band technique $^{9,10)}$. Although open surgical techniques are the standard method for reduction and fixation of displaced patellar fractures, Many reports describe the disadvantages and complications associated with traditional surgical treatments of patella fractures ${ }^{6,11)}$. The common complications of these techniques are: infection, loss of fixation, knee stiffness, posttraumatic osteoarthritis, nonunion, irritation of the anterior knee caused by internal fixation devices, and cosmetic problems ${ }^{6,11}$. Because the patella is a subcutaneous bone, fixation devices that have been used for fracture fixation are frequently irritating. Wires and wire knots seem to be particularly irritating to the soft tissues of the anterior knee area. In a report of a large series of patella fractures, the incidence of complications related to wire cerclage materials was $47 \%{ }^{4)}$. These complications resulted in a delay for patients in returning to their activities of daily living and a need for additional surgery. For this reason, new treatment modalities were researched to minimize these disadvantages. Some authors performed closed reduction and percutaneous fixation for patellar fractures ${ }^{12)}$. Luna-Pizarro et al. ${ }^{13)}$ showed that percutaneous osteosynthesis for displaced fractures of the patella was associated with shorter surgical time, better knee mobility, higher functional score, and lower complication rate than open surgery. Other authors reported their experience in arthroscopic-assisted fixation of patellar fractures. There are several advantages of arthroscopy in the treatment of patellar fractures: decompression of intra-articular hematomas for pain relief, removal and debridement of loose fragments, visualization of the articular surface for reduction. Even with open approach, arthroscopy has the merit of offering a better evaluation of articular congruity with magnified visual field ${ }^{14)}$.

Although the modified tension band wiring is still the most frequently applied internal fixation technique for transverse patella fractures, postoperative symptoms and complications related to this technique are not uncommon. A fragment displacement of $2 \mathrm{~mm}$ was observed in $22 \%-30 \%$ of the patients with early mobilization after tension band wiring ${ }^{12}$. Secondary postoperative pain due to skin irritation caused by the K-wires or the stainless steel wire is also a common problem which may require an extra surgery for fixation removal ${ }^{11)}$. Moreover, the "figure of 8 " tension band configuration was proved not the most stable construct for fixation of transverse patella fractures. Two separate studies $^{11,15)}$ have demonstrated through biomechanical evaluation that among the three methods for patella fixation (modified tension band, lag screws or cannulated screws with a tension band wired through the screws), the cannulated screws plus the tension band provided the most efficient stabilization, indicating that combining interfragmentary screw fixation with the tension band principle seems to provide improved stability over the modified tension band or screws alone for transverse patella fractures. Tian et al. ${ }^{16)}$ also showed that the cannulated screw tension band was better than the modified K-wire tension band technique. Thus this technique should be an effective device for fixation of transverse patella fractures.

\section{Conclusions}

Surgical treatment of transverse patella fractures with this percutaneous cannulated screws with tension band wiring technique, which combined the advantages of rigid internal fixation and minimally invasive surgery, has shown to provide satisfactory clinical results and excellent knee functions, with less pain and low incidence of complications. It could be a new option for treatment of transverse patella fractures.

\section{Conflict of Interest}

No potential conflict of interest relevant to this article was reported.

\section{References}

1. Lotke PA, Ecker ML. Transverse fractures of the patella. Clin Orthop Relat Res. 1981;(158):180-4.

2. Gosal HS, Singh P, Field RE. Clinical experience of patellar fracture fixation using metal wire or non-absorbable polyester: a study of 37 cases. Injury. 2001;32:129-35.

3. Catalano JB, Iannacone WM, Marczyk S, Dalsey RM, Deutsch LS, Born CT, Delong WG. Open fractures of the patella: long-term functional outcome. J Trauma. 1995;39:43944.

4. Hung LK, Chan KM, Chow YN, Leung PC. Fractured patella: operative treatment using the tension band principle. Injury. 1985;16:343-7. 
5. Appel MH, Seigel $\mathrm{H}$. Treatment of transverse fractures of the patella by arthroscopic percutaneous pinning. Arthroscopy. 1993;9:119-21.

6. Smith ST, Cramer KE, Karges DE, Watson JT, Moed BR. Early complications in the operative treatment of patella fractures. J Orthop Trauma. 1997;11:183-7.

7. Bostrom A. Fracture of the patella: a study of 422 patellar fractures. Acta Orthop Scand Suppl. 1972;143:1-80.

8. Tandogan RN, Demirors H, Tuncay CI, Cesur N, Hersekli M. Arthroscopic-assisted percutaneous screw fixation of select patellar fractures. Arthroscopy. 2002;18:156-62.

9. Carpenter JE, Kasman R, Matthews LS. Fractures of the patella. Instr Course Lect. 1994;43:97-108.

10. Kaylor KL. Injuries to the patella and extensor mechanism. In: Kasser JR, ed. Orthopaedic knowledge update 5. Rosemont, IL: American Academy of Orthopaedic Surgeons; 1996. p153-8.

11. Carpenter JE, Kasman RA, Patel N, Lee ML, Goldstein SA. Biomechanical evaluation of current patella fracture fixation techniques. J Orthop Trauma. 1997;11:351-6.
12. Leung PC, Mak KH, Lee SY. Percutaneous tension band wiring: a new method of internal fixation for mildly displaced patella fracture. J Trauma. 1983;23:62-4.

13. Luna-Pizarro D, Amato D, Arellano F, Hernandez A, LopezRojas P. Comparison of a technique using a new percutaneous osteosynthesis device with conventional open surgery for displaced patella fractures in a randomized controlled trial. J Orthop Trauma. 2006;20:529-35.

14. Chiang CC, Huang CK, Chen WM, Lin CF, Tzeng YH, Liu CL. Arthroscopically assisted percutaneous osteosynthesis of displaced transverse patellar fractures with figure-eight wiring through paired cannulated screws. Arch Orthop Trauma Surg. 2011;131:949-54.

15. Cekin T, Tükenmez M, Tezeren G. Comparison of three fixation methods in transverse fractures of the patella in a calf model. Acta Orthop Traumatol Turc. 2006;40:248-51.

16. Tian Y, Zhou F, Ji H, Zhang Z, Guo Y. Cannulated screw and cable are superior to modified tension band in the treatment of transverse patella fractures. Clin Orthop Relat Res. 2011;469:3429-35. 